\title{
Point: Impact of Prostate-Specific Antigen Velocity on Management Decisions and Recommendations
}

\author{
Stacy Loeb, MD, ${ }^{a}$ and $\mathrm{H}$. Ballentine Carter, $\mathrm{MD}^{\mathrm{b}}$
}

\begin{abstract}
Prostate-specific antigen (PSA) velocity predicts the presence of prostate cancer on biopsy and a greater risk of prostate cancer death after radical treatment. A new variation on PSA velocity called the risk count was recently shown to provide incremental reclassification for intermediate to high-grade disease on biopsy beyond PSA and age. These markers therefore have the potential to reduce overdiagnosis and overtreatment of indolent prostate cancer, and several professional guidelines support the use of PSA kinetics along with other predictors as part of the diagnostic algorithm. Among men already diagnosed with prostate cancer, PSA kinetics may also be helpful in predicting prognosis after definitive therapy. (JNCCN 2013;11:281-285)
\end{abstract}

Although prostate cancer screening with prostatespecific antigen (PSA) has been shown to reduce metastases and mortality from prostate cancer, ${ }^{1,2}$ its use has numerous drawbacks. A key issue is its limited specificity for clinically significant prostate cancer, resulting in unnecessary biopsies, overdiagnosis, and overtreatment. Fortunately, the way that screening is performed has evolved considerably over the past 2 decades, and there are several variations on the PSA test itself with improved performance characteristics.

One of these variations is PSA velocity (PSAV), which is a metric of the change in PSA level over time,

\footnotetext{
From the aDepartment of Urology, New York University, New York, New York, and bJames Buchanan Brady Urologic Institute, Johns Hopkins Medical Institutions, Baltimore, Maryland.

Submitted August 13, 2012; accepted for publication

January 28, 2013.

The authors have disclosed that they have no financial interests, arrangements, affiliations, or commercial interests with the manufacturers of any products discussed in this article or their competitors.

Correspondence: Stacy Loeb, MD, 550 1st Avenue, VZ30, 6th Floor (612), New York, NY 10016. E-mail: stacyloeb@gmail.com
}

and has been known since the early 1990s to increase the specificity of PSA-based screening for prostate cancer detection. ${ }^{3}$ This is important because prostate biopsies have potential associated risks, ${ }^{4}$ such that reducing false-positive screening tests is an important objective. Among men aged 50 years and younger without prostate cancer, the median PSAV is approximately 0.00 to $0.03 \mathrm{ng} / \mathrm{mL} / \mathrm{y} .{ }^{5,6}$ Recent studies have suggested that the baseline PSAV may be useful for risk stratification in young men. ${ }^{7}$ At the other end of the spectrum, PSAV may also be useful in guiding the discontinuation of screening in elderly men. ${ }^{8,9}$

Another area in which improvements are needed is distinguishing indolent from aggressive disease. A large body of evidence now shows a strong relationship between PSAV and more aggressive prostate cancer features. For example, in 1073 men from a large radical prostatectomy series, insignificant tumors were present in $10 \%$ of men with a PSAV less than $0.4 \mathrm{ng} / \mathrm{mL} / \mathrm{y}$, and in only $5 \%$ of men with a PSAV greater than 0.4 $\mathrm{ng} / \mathrm{mL} / \mathrm{y} .{ }^{10}$ These results suggested that PSAV may be a marker for clinically significant prostate cancer. Another recent study of 219,388 US men from Kaiser Permanente showed that the annual percent change in PSA was significantly better than the total PSA for discriminating high-grade prostate cancer on biopsy (area under the receiver operating curve of 0.955 vs. 0.727 , respectively). ${ }^{11}$

PSAV has also been shown to predict prostate cancer-specific mortality. In a landmark study, D'Amico et $\mathrm{al}^{12}$ reported that a PSAV greater than $2 \mathrm{ng} / \mathrm{mL} / \mathrm{y}$ predicted a greater risk of prostate cancer death after radical prostatectomy. These authors subsequently validated the relationship between a PSAV greater than $2 \mathrm{ng} / \mathrm{mL} / \mathrm{y}$ and the risk of mortality after radiation therapy. ${ }^{13}$ The independent relationship between PSAV and long-term treatment outcomes was also 
demonstrated by Sengupta et al. ${ }^{14}$ These studies suggest that PSAV identifies a group that is at higher risk of ultimate disease progression and death, despite curative therapy.

Subsequently, Carter et $\mathrm{al}^{15}$ showed that men with a PSAV greater than $0.35 \mathrm{ng} / \mathrm{mL} / \mathrm{y}$, even at a time when the total PSA was low, had a 5-fold increased risk of prostate cancer death more than a decade later. Thus, PSAV also has future prognostic value with regard to the likelihood of lethal prostate cancer.

Conversely, other studies have failed to show an "important" relationship between PSAV and prostate cancer-related outcomes. For example, Vickers et al ${ }^{16}$ performed a comprehensive systematic review of studies on PSA kinetics published before March 2007, concluding that the existing evidence did not support the utility of PSA kinetics in clinical decision-making for early-stage prostate cancer.

Several reasons exist for these seemingly divergent results. First, the systematic review by Vickers et $\mathrm{al}^{16}$ included heterogeneous studies of PSA kinetics both before and after a prostate cancer diagnosis to predict diverse outcomes ranging from biopsy outcome to progression on active surveillance. Studies have since shown that PSA kinetics may be more useful in certain settings than in others. For example, in the Johns Hopkins active surveillance program, PSAV (and PSA doubling time) were not reliably indicative of disease reclassification on biopsy, ${ }^{17}$ whereas other programs have reported conflicting results. ${ }^{18}$ Currently, a lack of consensus exists regarding the use of PSA kinetics to indicate progressive disease in active surveillance programs. ${ }^{19}$ In the systematic review by Vickers et al, ${ }^{16}$ combining disparate outcomes such as initial biopsy results and progression on active surveillance in a systematic review may have affected the results.

Furthermore, the systematic review included PSAV along with other types of PSA kinetics measurements involving different mathematical assumptions (eg, PSA doubling time). ${ }^{20}$ However, this authors' group showed that PSAV is more useful than PSA doubling time in both the diagnostic setting ${ }^{21}$ and the prediction of treatment outcomes, ${ }^{22}$ indicating that grouping these different measurements together is inappropriate.

Perhaps the most important consideration is that several of the early studies reporting a robust association between PSAV and prostate cancer aggressiveness did not address the accuracy and predictive value of PSAV beyond that offered by total PSA and other standard predictors. In fact, the systematic review by Vickers et $\mathrm{al}^{16}$ found that among the 87 eligible articles, only a single study reported an increase in predictive accuracy using PSA kinetics. In fact, this particular study, which was published by this article's authors' group, ${ }^{23}$ did show an improvement in predictive accuracy with the addition of PSA kinetics to a model with race, PSA, age, and family history (area under the curve [AUC], 0.83 vs. 0.81), but was criticized for verification bias. Thus, at the time of the systematic review by Vickers et $\mathrm{al}^{16}$ there were numerous gaps in the evidence regarding PSAV, which hampered conclusions. Fortunately, the evidence base and statistical methodology have expanded substantially since 2007.

In one of the major studies to emerge in the past 5 years, Vickers et a $^{24}$ examined PSAV in 5519 participants from the Prostate Cancer Prevention Trial (PCPT). On multivariable analysis, they showed that PSAV was associated with a 5.2-fold increased risk of prostate cancer detection on biopsy. Furthermore, they reported on predictive accuracy using receiver operating characteristic (ROC) analysis. When added to a base model with log PSA, family history, digital rectal examination findings, and prior biopsy history, PSAV increased the AUC for the discrimination of all end points: overall cancer, clinically significant cancer, and Gleason 7 to 10 disease.

An advantage of this population from the PCPT is that empiric biopsies were performed as part of the protocol in the absence of a clinical indication, providing histologic confirmation for all participants. However, drawbacks are that the population was elderly ( $79 \%$ were age $\geq 65$ years) and had been serially screened for at least 7 years, resulting in a population enriched with indolent disease. From an analytic standpoint, a limitation of this study is the reliance on ROC analysis, which has been shown to be insensitive to the incremental predictive value of new markers. ${ }^{25,26}$ Additionally, as pointed out by Vickers et al, ${ }^{16}$ it is "naturally difficult for a marker to add value to a predictor with which it has a strong correlation," as is the case with total PSA and PSAV. Despite these limitations, the authors stated a strong 
conclusion that decisions should be based on PSA alone and that PSAV is not worth the incremental time or expense.

As this article's authors previously summarized in an editorial comment, ${ }^{27}$ the synopsis by Vickers et $\mathrm{al}^{16,24}$ seems to suggest little reason to maintain PSA values in the medical record. However, patients frequently present to the urology clinic with a listing of prior PSA data, and disregarding historical information is not a practicable option in the realworld clinical setting. Therefore, the real question is not whether to discard or consider the prior values, but how to make the best use of these data when available. Current evidence supports that the answer to this question is a new concept called PSAV risk count. ${ }^{28}$

This concept was initially proposed by Carter et $\mathrm{al}^{29}$ in men from the Baltimore Longitudinal Study of Aging (BLSA). For men with several serial PSA values, the first step is to calculate 2 PSAV measurements in a row. As the name suggests, the "risk count" then involves counting the number of times in a row that the PSAV exceeds a specific threshold (eg, $0.4 \mathrm{ng} / \mathrm{mL} / \mathrm{y}$ ). Risk counts of 0,1 , and 2 indicate that neither, one, or both PSAV measurements are greater than $0.4 \mathrm{ng} / \mathrm{mL} / \mathrm{y}$, respectively. The initial study in the BLSA showed that the probability of high-risk prostate cancer increased significantly with increasing PSAV risk count. ${ }^{29}$ More recently, the study by Vickers et $\mathrm{al}^{24}$ in the PCPT similarly showed that the number of times the PSA level increased to greater than $0.4 \mathrm{ng} / \mathrm{mL} / \mathrm{y}$ was a significant predictor of overall prostate cancer detection on biopsy, but they did not assess the relationship of risk count to aggressive disease.

Finally, the utility of PSAV risk count was recently externally validated in a larger independent population of 18,214 men from an established screening cohort. ${ }^{30}$ After adjusting for age and PSA, men with a risk count of 2 had an 8-fold increased risk of prostate cancer diagnosis and a 5.4-fold increased risk of Gleason 8 to 10 disease on biopsy. On ROC analysis, PSAV risk count significantly improved predictive accuracy for overall prostate cancer compared with a base model of age and PSA alone $(P=.026)$. It also led to a significant increase in the discrimination of Gleason 8 to 10 disease (AUC, 0.725 with risk count plus age and PSA vs. 0.625 for age and PSA alone; $P=.031$ ). As discussed earlier, ROC analysis has limitations in evaluating new markers. ${ }^{26}$ Thus, as in major studies from other disciplines, ${ }^{31}$ net reclassification analysis was also used to evaluate risk count and high-grade disease. For this analysis, 2 different definitions of high-grade disease were used: Gleason 7 to 10 (which spans a more heterogeneous group but includes a larger number of events) and Gleason 8 to 10 (the most lifethreatening group but is limited by a smaller number of events). Regardless of the definition, PSAV risk count led to a statistically and clinically significant reclassification in the risk of Gleason 7 or greater $(z=6.5 ; P<.001)$ and Gleason 8 or greater disease on biopsy $(z=3.5 ; P<.001)$, when compared with age and PSA. ${ }^{30}$ These results suggest that, compared with age and PSA alone, PSAV risk count may be useful to reduce unnecessary biopsies and the diagnosis of low-risk prostate cancer. Correspondingly, PSAV risk count was recently added as a talking point in the 2012 NCCN Clinical Practice Guidelines in Oncology (NCCN Guidelines) for Prostate Cancer Early Detection (to view the most recent version of these guidelines, visit NCCN.org). ${ }^{32}$

Despite this robust evidence supporting PSAV, several important considerations exist. As with a single PSA measurement, confounding of PSAV is possible. For example, studies from the United States and Europe have shown that sudden high spikes in PSA that impact PSAV often reflect prostatitis rather than prostate cancer. ${ }^{33,34}$ This is where the art of medicine and clinical judgement play a key role. Park et $\mathrm{al}^{35}$ recently showed how recalculating PSAV after eliminating confounding factors reduced false-positive and false-negative results. Although these considerations form a routine part of judicious clinical practice when making decisions regarding biopsy and treatment, retrospective analyses of PSA kinetics in existing large-scale databases may show less favorable results by ignoring these patientspecific factors. Certainly, these issues highlight the sensibility of using a calculation such as PSAV risk count, which helps to single out the individuals with sustained PSA increases with a higher risk for lifethreatening disease. . $^{29,30}$

Additionally, no randomized trial has ever compared the outcomes of PSAV- or risk count-based screening versus traditional screening approaches. In randomized screening trials, such as the Prostate, Lung, Colorectal, and Ovarian (PLCO) cancer 
screening trial, ${ }^{36}$ European Randomized Study of Screening for Prostate Cancer (ERSPC), ${ }^{1}$ and the Goteborg population-based randomized screening trial, ${ }^{2}$ biopsy recommendations were based on total PSA levels rather than PSA kinetics. This article's authors believe that PSAV may help maximize the benefits of screening (ie, early detection of lifethreatening disease, leading to reduced morbidity and mortality) and reduce the harms (ie, unnecessary biopsies, overdiagnosis, and overtreatment of insignificant disease); however, this concept has not been tested in a randomized fashion, and such a trial is unlikely to occur.

Although additional observational evidence continues to accumulate on the incremental value of PSAV and risk count, the bulk of contemporary data supports their clinical utility. Therefore, PSAV has been incorporated into several screening guidelines. The 2012 NCCN Guidelines for Prostate Cancer Early Detection recommend considering a biopsy for a suspicious digital rectal examination, PSA level greater than 2.5, or PSAV of $0.35 \mathrm{ng} / \mathrm{mL} / \mathrm{y}$ or greater, and also discuss the use of PSAV risk count as a talking point. ${ }^{32}$ In the best practice statement on PSA, the American Urological Association also recommends PSAV among the factors that should be considered along with PSA in determining the need for biopsy. ${ }^{37}$ For men diagnosed with prostate cancer, PSAV should also be used in risk stratification and the assessment of recurrence risks after treatment.

\section{References}

1. Schroder FH, Hugosson J, Roobol MJ, et al. Prostate-cancer mortality at 11 years of follow-up. N Engl J Med 2012;366:981990.

2. Hugosson J, Carlsson S, Aus G, et al. Mortality results from the Goteborg randomised population-based prostate-cancer screening trial. Lancet Oncol 2010;11:725-732.

3. Carter HB, Pearson JD, Metter EJ, et al. Longitudinal evaluation of prostate-specific antigen levels in men with and without prostate disease. JAMA 1992;267:2215-2220.

4. Loeb S, Carter HB, Berndt SI, et al. Complications after prostate biopsy: data from SEER-Medicare. J Urol 2011;186:1830-1834.

5. Tang P, Du W, Xie K, et al. Characteristics of baseline PSA and PSA velocity in young men without prostate cancer: racial differences. Prostate 2012;72:173-180.

6. Loeb S, Roehl KA, Catalona WJ, et al. Is the utility of prostatespecific antigen velocity for prostate cancer detection affected by age? BJU Int 2008;101:817-821.

7. Sun L, Moul JW, Hotaling JM, et al. Prostate-specific antigen (PSA) and PSA velocity for prostate cancer detection in men aged $<50$ years. BJU Int 2007;99:753-757.
8. Tang P, Sun L, Uhlman MA, et al. Prostate-specific antigen velocity based risk-adapted discontinuation of prostate cancer screening in elderly men. BJU Int 2011;108:44-48.

9. Mouraviev V, Broadwater G, Sun L, et al. Can prostate-specific antigen and prostate-specific antigen velocity be used for prostate cancer screening in men older than 70 years? Urology 2008; 71:1020-1023.

10. Loeb S, Roehl KA, Helfand BT, et al. Can prostate specific antigen velocity thresholds decrease insignificant prostate cancer detection? J Urol 2010;183:112-116.

11. Wallner LP, Frencher SK, Hsu JW, et al. Changes in serum prostatespecific antigen levels and the identification of prostate cancer in a large managed care population. BJU Int, in press.

12. D'Amico AV, Chen MH, Roehl KA, et al. Preoperative PSA velocity and the risk of death from prostate cancer after radical prostatectomy. N Engl J Med 2004;351:125-135.

13. D'Amico AV, Renshaw AA, Sussman B, et al. Pretreatment PSA velocity and risk of death from prostate cancer following external beam radiation therapy. JAMA 2005;294:440-447.

14. Sengupta S, Myers RP, Slezak JM, et al. Preoperative prostate specific antigen doubling time and velocity are strong and independent predictors of outcomes following radical prostatectomy. J Urol 2005;174:2191-2196.

15. Carter HB, Ferrucci L, Kettermann A, et al. Detection of lifethreatening prostate cancer with prostate-specific antigen velocity during a window of curability. J Natl Cancer Inst 2006;98:15211527.

16. Vickers AJ, Savage C, O'Brien MF, et al. Systematic review of pretreatment prostate-specific antigen velocity and doubling time as predictors for prostate cancer. J Clin Oncol 2009;27:398-403.

17. Ross $\mathrm{AE}$, Loeb $\mathrm{S}$, Landis $\mathrm{P}$, et al. Prostate-specific antigen kinetics during follow-up are an unreliable trigger for intervention in a prostate cancer surveillance program. J Clin Oncol 2010;28:28102816.

18. Klotz L, Zhang L, Lam A, et al. Clinical results of long-term followup of a large, active surveillance cohort with localized prostate cancer. J Clin Oncol 2010;28:126-131.

19. Iremashvili $\mathrm{V}$, Manoharan M, Lokeshwar SD, et al. Comprehensive analysis of post-diagnostic prostate-specific antigen kinetics as predictor of a prostate cancer progression in active surveillance patients. BJU Int, in press.

20. Carter HB. Differentiation of lethal and non lethal prostate cancer: PSA and PSA isoforms and kinetics. Asian J Androl 2012;14:355360.

21. Loeb S, Kettermann A, Ferrucci L, et al. PSA doubling time versus PSA velocity to predict high-risk prostate cancer: data from the Baltimore Longitudinal Study of Aging. Eur Urol 2008;54:10731080.

22. Loeb S, Kan D, Yu X, et al. Preoperative prostate specific antigen doubling time is not a useful predictor of biochemical progression after radical prostatectomy. J Urol 2010;183:1816-1821.

23. Loeb S, Roehl KA, Catalona WJ, et al. Prostate specific antigen velocity threshold for predicting prostate cancer in young men. J Urol 2007;177:899-902.

24. Vickers AJ, Till C, Tangen CM, et al. An empirical evaluation of guidelines on prostate-specific antigen velocity in prostate cancer detection. J Natl Cancer Inst 2011;103:462-469.

25. Pencina MJ, D’Agostino RB Sr, D’Agostino RB Jr, et al. Evaluating the added predictive ability of a new marker: from area under the ROC curve to reclassification and beyond. Stat Med 2008;27:157- 
Point: PSA Velocity in Prostate Cancer Risk Stratification

172.

26. Cook NR. Use and misuse of the receiver operating characteristic curve in risk prediction. Circulation 2007;115:928-935.

27. Loeb S, Metter EJ, Carter HB. Re: an empirical evaluation of guidelines on prostate-specific antigen velocity in prostate cancer detection. J Natl Cancer Inst 2011;103:1636-1637.

28. Fillon M. Study supports PSA velocity risk count. J Natl Cancer Inst 2012;104:1042-1043.

29. Carter HB, Kettermann A, Ferrucci L, et al. Prostate-specific antigen velocity risk count assessment: a new concept for detection of life-threatening prostate cancer during window of curability. Urology 2007;70:685-690.

30. Loeb S, Metter EJ, Kan D, et al. Prostate-specific antigen velocity (PSAV) risk count improves the specificity of screening for clinically significant prostate cancer. BJU Int 2012;109:508-513.

31. Inker LA, Schmid CH, Tighiouart $\mathrm{H}$, et al. Estimating glomerular filtration rate from serum creatinine and cystatin C. N Engl J Med 2012;367:20-29.

32. Presti JC, Andriole G, Bahnson RR, et al. NCCN Clinical Practice
Guidelines in Oncology: Prostate Cancer Early Detection. Version 2, 2012. Available at NCCN.org. Accessed June 12, 2012.

33. Eggener SE, Yossepowitch O, Roehl KA, et al. Relationship of prostate-specific antigen velocity to histologic findings in a prostate cancer screening program. Urology 2008;71:1016-1019.

34. Vickers AJ, Wolters T, Savage CJ, et al. Prostate-specific antigen velocity for early detection of prostate cancer: result from a large, representative, population-based cohort. Eur Urol 2009;56:753760.

35. Park JJ, Chen MH, Loffredo M, et al. Prostate-specific antigen velocity before and after elimination of factors that can confound the prostate-specific antigen level. Int J Radiat Oncol Biol Phys 2012;82:1217-1221.

36. Andriole GL, Crawford ED, Grubb RL III, et al. Prostate cancer screening in the randomized Prostate, Lung, Colorectal, and Ovarian Cancer Screening Trial: mortality results after 13 years of follow-up. J Natl Cancer Inst 2012;104:125-132.

37. Greene KL, Albertsen PC, Babaian RJ, et al. Prostate specific antigen best practice statement: 2009 update. J Urol 2009;182:2232-2241. 